\title{
Infants' Neural Responses to Helping and Hindering Scenarios
}

\author{
Enda Tan a and J. Kiley Hamlin ${ }^{\mathrm{a}}$ \\ ${ }^{a}$ University of British Columbia, 2136 West Mall, Vancouver, BC, Canada
}

Correspondence concerning this paper should be addressed to Enda Tan, Department of

Psychology, University of British Columbia, 2136 West Mall, Vancouver, BC, Canada V6T $1 Z 4$. E-mail: enda.tan@psych.ubc.ca

Running title: INFANTS' NEURAL RESPONSES TO SOCIOMORAL SCENES 


\begin{abstract}
A growing literature suggests infants prefer prosocial others over antisocial others. Although recent studies have begun to explore the neural mechanisms underlying these responses (Cowell \& Decety, 2015; Gredebäck et al., 2015), these studies were based on relatively small samples and focused on distinct aspects of sociomoral responding. The current preregistered study systematically examined infants' neural responses both to prosocial/antisocial interactions and to prosocial/antisocial characters, using larger samples and two distinct age groups. We found that 6- (but not 12-) month-olds showed higher relative right frontal alpha power (indexing approach motivation) when viewing helping versus hindering scenarios. Consistent with past EEG work, infants showed no group-level manual preferences for the helper. However, analyses of infants' neural responses toward images of the helper versus hinderer revealed that both 6- and 12-month-olds showed differential event-related potential (ERP) responses in the P400 and N290 components (indexing social perception) but not in the Nc component (indexing attentional allocation), suggestive that infants' neural responses to prosocial versus antisocial characters reflect social processing. Together, these findings provide a more comprehensive account of infants' responses to prosocial/antisocial interactions and characters, and support the hypothesis that both motivational and socially relevant processes are implicated in infants' sociomoral responding.
\end{abstract}

Keywords: infant, EEG, sociomoral evaluation, frontal alpha asymmetry, P400/N290, Nc 


\section{Introduction}

Evolutionary theories suggest that natural selection favors the early emergence of sociomoral evaluation in humans (Hrdy, 1999; Joyce, 2006; Sheskin et al., 2014). Consistent with this possibility, a growing literature suggests that even preverbal infants are sensitive to sociomoral interactions between novel third parties (for review and meta-analysis, see Margoni \& Surian, 2018). For example, in one line of research, infants viewed live puppet shows in which a climber character tried repeatedly but unsuccessfully to reach the top of a steep hill, and was subsequently helped (pushed uphill) or hindered (pushed downhill) by two other characters. When presented with both the helpful and unhelpful characters, 6-10-month-olds directed their first touch to the helper rather than the hinderer (Hamlin, 2015; Hamlin et al., 2007), and 3- and 6-month-olds looked longer at the helper than the hinderer (Hamlin et al., 2010). Together, these results suggested that preverbal infants may engage in rudimentary sociomoral evaluation.

Besides the "hill” scenario, infants' preferences for prosocial versus antisocial characters have been found using scenarios depicting other types of prosocial/antisocial interactions (Buon et al., 2014; Geraci \& Surian, 2011; Hamlin \& Wynn, 2011; Scola et al., 2015; for review, see Holvoet et al., 2016; Margoni \& Surian, 2018); these findings provide evidence that infants' preferences for prosocial others are robust. Recently, there has also been an increasing interest in clarifying the mechanisms underlying infants' responses to sociomoral scenes. Using behavioral paradigms, researchers have begun to illuminate whether infants' responses to sociomoral scenes are driven by social versus perceptual features of the displays (Hamlin, 2015; Scarf et al., 2012; Tan \& Hamlin, 2021), whether these responses are based on the intentions versus outcomes of prosocial/antisocial actions (Hamlin, 2013; Woo et al., 2017), and whether and how affective processes are involved (Steckler et al., 2018). 
Although behavioral research has provided insights into the mental processes underlying infants' sociomoral responding, another fruitful way of probing these processes would be to examine infants' neural activity as they view prosocial/antisocial events and characters. Specifically, neuroimaging techniques such as electroencephalography (EEG) allow researchers to better elucidate the nature of mental processes via distinct neural signatures, as well as to delineate the time course of mental events. To date, only two studies have examined infants' neural responses to helping/hindering scenarios (Cowell \& Decety, 2015; Gredebäck et al., 2015); these two studies focused on distinct aspects of sociomoral responding.

In one study, researchers assessed 12- to 24-month-old infants' EEG responses while they viewed helping/hindering events in the hill scenario (Cowell \& Decety, 2015). They found that infants showed greater left (vs. right) frontal alpha power when viewing hindering (vs. helping) scenarios. Crucially, greater left frontal alpha power has been associated with avoidance/withdrawal motivation, whereas greater right frontal alpha power has been associated with approach motivation (for review, see Harmon-Jones \& Gable, 2018; Reznik \& Allen, 2018). The findings of Cowell and Decety (2015) therefore suggest that antisocial (vs. prosocial) interactions elicit more avoidance/withdrawal motivation in infants, consistent with behavioral findings that infants are likely to subsequently avoid antisocial characters during a choice procedure (Hamlin, 2015; Hamlin et al., 2007).

In another study, researchers explored whether socially relevant neural mechanisms are implicated in infants' responses to characters who had previously acted prosocially or antisocially (Gredebäck et al., 2015). They first familiarized 6-month-olds with helping/hindering hill events, and subsequently examined infants' event-related potential (ERP) responses to still images of the helper and hinderer. Infants showed differential ERP responses to 
helper/hinderer images in the P400 component, thought to be a neural correlate of social perception in infants (Gredebäck \& Daum, 2015; Gredebäck et al., 2015), but not in the Nc component, thought to index domain-general, attention-related processing (Csibra et al., 2008; Luyster et al., 2014; Nelson \& Monk, 2001). The findings of Gredebäck et al. (2015) therefore suggest that infants' responses to helpful/unhelpful characters may selectively recruit socially relevant neural mechanisms.

The two studies reviewed above provide preliminary evidence that infants' responses to helping/hindering interactions and characters involve approach-avoidance motivations and socially relevant processes, offering crucial insights into the mechanisms underlying infants' sociomoral responding. That said, these results were based on relatively small samples $(\mathrm{N}=14$ per experiment in Gredebäck et al., 2015; N=25 in Cowell \& Decety, 2015) and one included toddlers rather than infants and a wide age range (12 to 24 months; Cowell \& Decety, 2015). Thus, it is necessary to assess the robustness of these effects with larger and more age-specific samples. Moreover, these two studies focused on distinct aspects of sociomoral responding (i.e., neural responses to prosocial/antisocial interactions versus prosocial/antisocial characters) and used different EEG measures. To provide a more comprehensive picture of infants' sociomoral responding, it is important to analyze neural processes both during prosocial/antisocial events, and to prosocial/antisocial characters after the events occur.

The current preregistered study aimed to improve upon past research by systematically examining infants' EEG responses to both prosocial/antisocial interactions and characters, using larger samples, across two age groups, combining all measures used in prior studies.

Specifically, we familiarized both 6- and 12-month-old infants (total $\mathrm{N}=74$ ) with hill helping and hindering events, and examined their frontal alpha responses during helping/hindering as well as 
their ERP (e.g., P400, Nc) responses to images of the helper/hinderer. In order to better understand the dynamics of infants' neural activity throughout helping and hindering events, we divided the scenarios into different phases and examined frontal alpha responses during each phase. To investigate whether infants' responses to helpers versus hinderers recruit socially relevant processes, we examined whether helper/hinderer images elicit differential ERP responses in socially relevant components (e.g., P400) versus a more domain-general, attentionrelated component (Nc). Finally, following Cowell and Decety (2015), we examined whether infants' neural responses to prosocial/antisocial scenarios predict manual preferences toward the helper, to potentially shed light on the links between neural and behavioral responses. That said, we did not expect infants to show a group-level preference for the helper (vs. hinderer), because past studies in which infants wore EEG caps (Cowell \& Decety, 2015) and in which a familiarization (vs. habituation) procedure was used (Schlingloff et al., 2020) did not find a group-level helper preference.

\section{Materials and Methods}

\subsection{Participants}

Participants were 42 6-month-old infants (22 females; mean age=6.12 months, range $=5.50-6.63$ months $)$ and 32 12-month-old infants (16 females; mean age $=11.96$ months, range $=11.50-12.53$ months) living in a North American city. These two age groups were selected based on Gredebäck et al. (2015) (6-month-olds) and Cowell and Decety (2015) (12- to 24-month-olds) to facilitate data comparison across studies. All participants were full-term and healthy. Most participants were from Caucasian and Asian backgrounds, representative of the population in the area. For the 6-month group, an additional 33 infants were excluded because 
they did not meet the preregistered inclusion criteria (see details below) due to fussiness ( $N=20$ ), inattentiveness $(\mathrm{N}=7)$, technical errors $(\mathrm{N}=5)$, and parental interference $(\mathrm{N}=1)$. For the 12-month group, an additional 32 infants were excluded due to fussiness $(\mathrm{N}=23)$, inattentiveness $(\mathrm{N}=6)$, technical errors $(\mathrm{N}=1)$, and parental interference $(\mathrm{N}=2)$. These attrition rates $(44 \%$ for 6 -montholds and $50 \%$ for 12 -month-olds) are similar to those reported in past ERP study using the hill paradigm (43\% for 6-month-olds in Gredebäck et al., 2015) and in infant ERP studies in general (49.16\% according to a meta-analysis; Stets et al., 2012).

The target sample size ( $\mathrm{N}=32$ per age group) was based on past $\mathrm{EEG}(\mathrm{N}=14$ in Gredebäck et al., 2015; $\mathrm{N}=25$ in Cowell \& Decety, 2015) and behavioral (N=16 in Hamlin et al., 2007; N=24 in Hamlin, 2015) studies using the hill paradigm, and increased to 32 to improve statistical power. We ended up with 10 additional participants for the 6-month group because we kept recruiting until we had recruited enough for both age groups. Because 12-month-olds were harder to recruit, by the time we stopped recruiting for the whole project we had more participants in the 6-month group. The decision to include these participants was made prior to analysis. The target sample size, inclusion criteria, procedure, and analysis plan were preregistered on Open Science Framework (OSF); deviations from preregistration are described and justified (see Supplementary Material; SM 1-2). The study was conducted in accordance with the ethical standards of the American Psychological Association, and data were collected with approval from the university's Research Ethics Board.

\subsection{Procedure and Stimuli}

The stimuli were presented on a 23.6" LED screen (Samsung S24B300HL; width: 1920 pixels, $22.4^{\prime \prime}, 51^{\circ}$ visual angle; height: 1080 pixels, $13.6^{\prime \prime}, 32^{\circ}$ visual angle). Infants sat on their 
parents' lap approximately $60 \mathrm{~cm}$ from the computer screen in a sound-attenuated booth. Parents were instructed to refrain from distracting infants and to close their eyes throughout the procedure. Stimulus presentation was controlled by an experimenter outside the booth using EPrime 2.0 (Psychology Software Tools Inc., Pittsburgh, PA). Infants' behaviors were videotaped and monitored throughout the study and coded offline after the study. Event markers were added to EEG signals when infants looked away from the screen and looked back to the screen, and when major body movements (e.g., crying, grabbing the EEG cap) started and ended.

Familiarization and test trials were grouped into blocks. Each block consisted of 3 helping and 3 hindering videos (familiarization trials) presented in a counterbalanced order, followed by 20 helper and 20 hinderer images (test trials) presented in random order (see Figure 1). Each familiarization trial consisted of a "ding" sound (1000 ms), a black screen (3000 ms), and a helping or hindering video (16800 ms), followed by a black screen. Each test trial consisted of an attention getter (1000 ms), a fixation cross (jittered between 1200-1400 ms), and an image showing the helper or hinderer against hill background (2000 ms), followed by a black screen. To ensure infants were attentive before trials began, the experimenter manually advanced each trial from the black screen.

Helping/hindering videos were created using Blender (www.blender.org; see Figure 2) and depicted a climber's attempts to get to the top of a steep hill. The climber (a red circle with eyes; diameter $=4^{\circ}$ visual angle) successfully climbed the first half of the hill $(0-2000 \mathrm{~ms})$ and attempted twice to climb the (steeper) second half of the hill, falling down each time (2000$9000 \mathrm{~ms}$ ). On their third attempt, either a helper or a hinderer (a blue square or yellow triangle with eyes; diameter $\approx 4^{\circ}$ visual angle) entered the scene. The helper entered from the bottom of the hill (9000-9500ms), pushed the climber to the top of the hill (facilitating their goal; 9500- 
$13000 \mathrm{~ms})$, and left the scene from the bottom of the hill (13000-16800ms). The hinderer entered from the top of the hill (9000-9500ms), pushed the climber to the bottom of the hill (hindering their goal; 9500-13000ms), and left the scene from the top of the hill (13000-16800ms). The order of helping and hindering videos and the color/shape of the helper and the hinderer (blue square/yellow triangle) were counterbalanced across participants. The climber's eyes were fixated at the hilltop during their attempts, and the helper/hinderer's eyes were fixated at the climber. 


\section{Familiarization Trial}

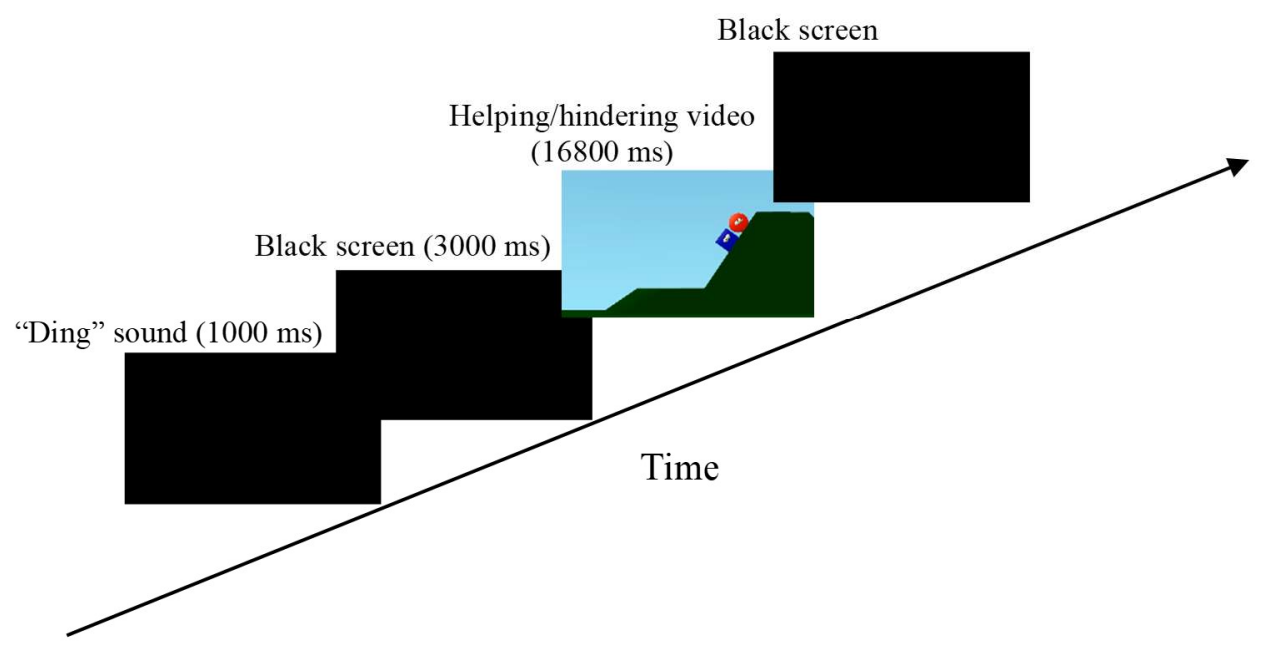

\section{Test Trial}

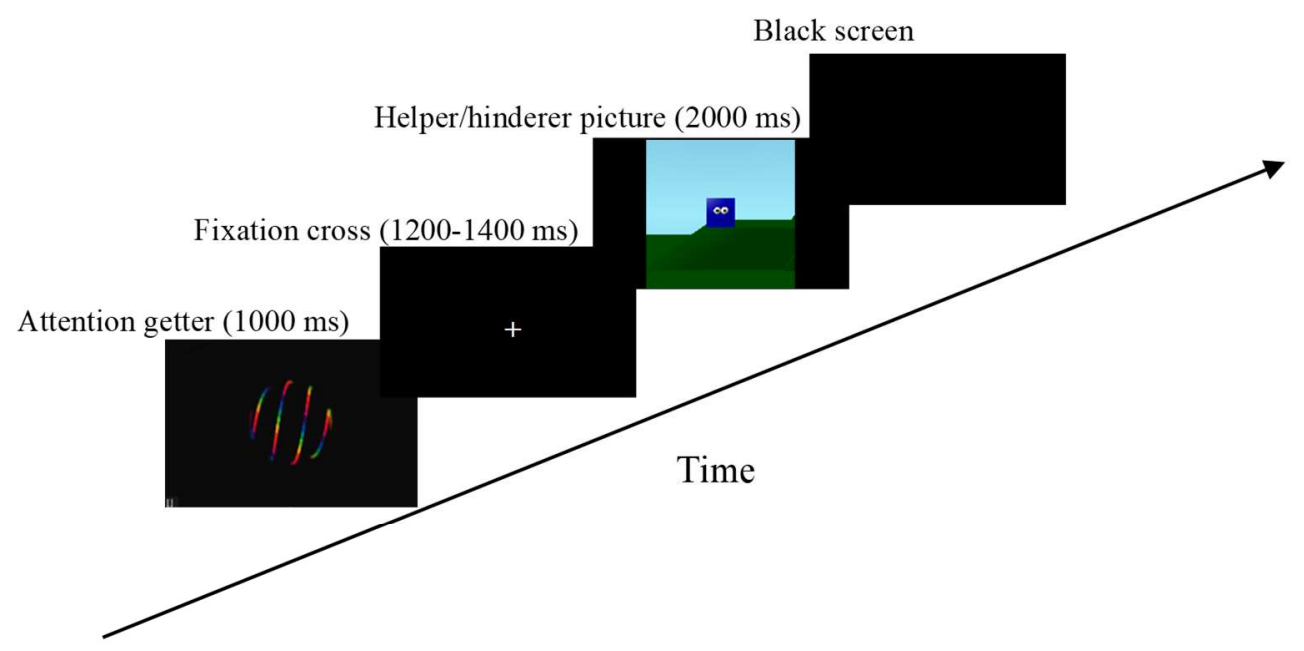

Figure 1. Schematic diagram of the stimuli. 

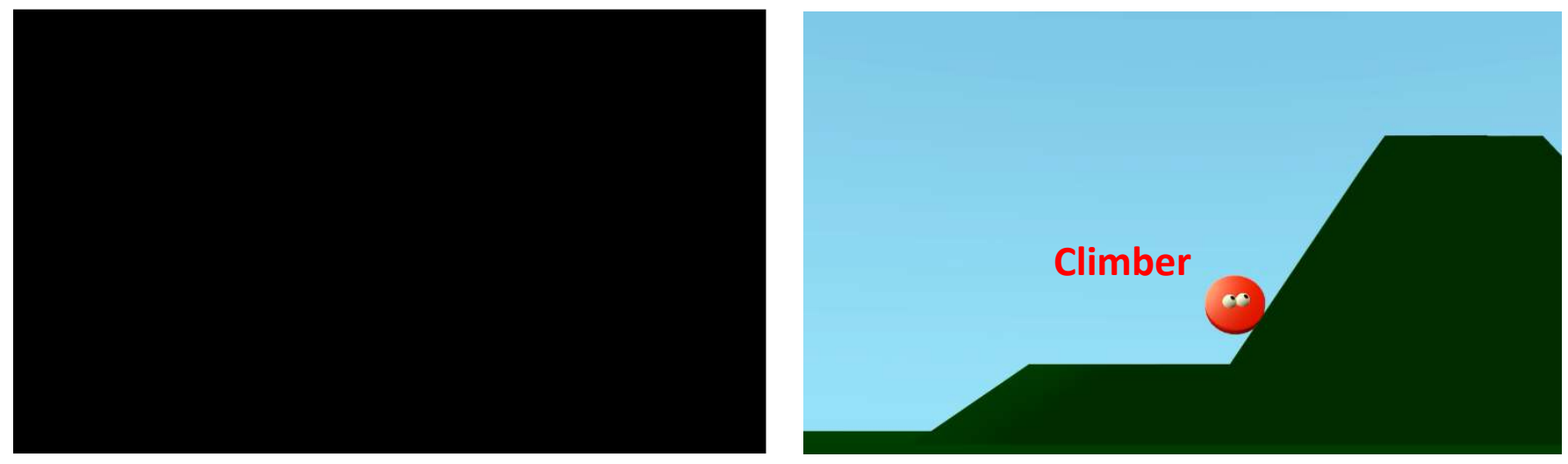

Baseline (-2000 - 0ms)

$$
\text { Pre-phase (0 - 9500ms) }
$$
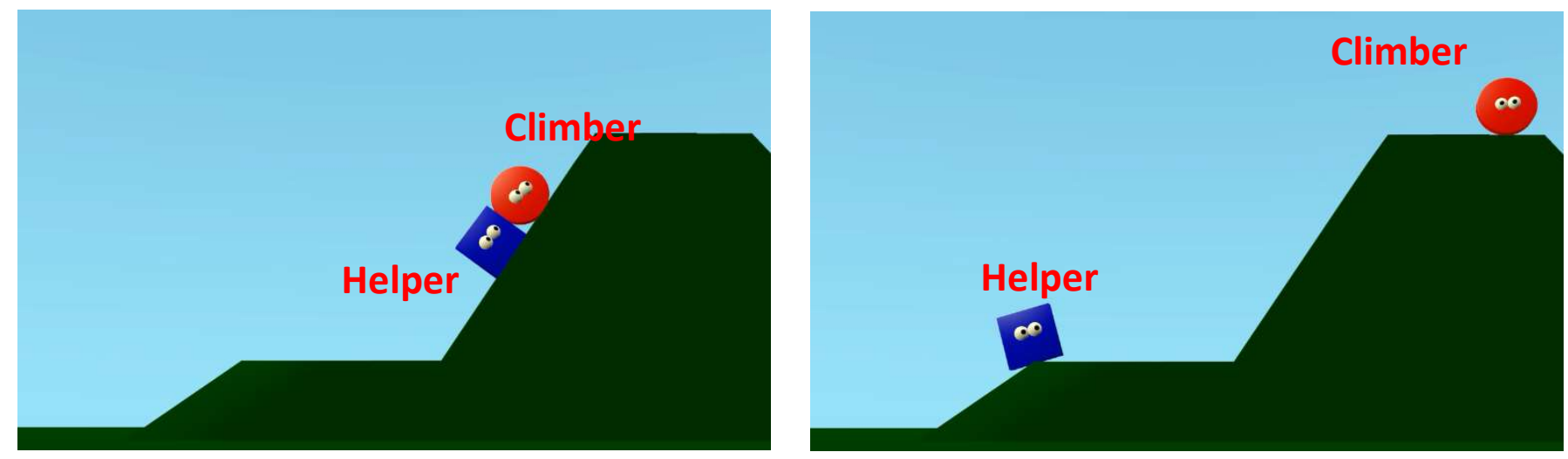

During-phase (9500 - 14000ms)

Post-phase (14000 - 16800ms)

Figure 2. Phases of familiarization trials. Note that the identity of the helper/hinderer was counterbalanced across participants. 
Participants first viewed one block of familiarization trials and test trials. Once all the stimuli were presented, or if infants became inattentive during test trials (i.e., they lost interest in viewing still images), another block of familiarization trials (helping/hindering videos) and test trials was presented. The stimuli were repeated until infants stopped attending and/or became fussy; the average number of blocks presented was 2.00 for 6-month-olds and 2.09 for 12-montholds. Then the manual choice task began. The experimenter entered the booth, presented the infants with paper cut-outs of the helper and the hinderer placed $25 \mathrm{~cm}$ apart on a white board, and asked "would you like to play with one?" Infants' choice was defined as the first character picked by infants through a visually guided touch.

As per preregistered inclusion criteria, participants in the final sample had 1) viewed at least 3 helping and 3 hindering videos, 2) provided at least 60 artifact-free segments for familiarization trial frontal alpha asymmetry analysis (Cowell \& Decety, 2015; see details below), and 3) provided at least 10 artifact-free trials for helper and hinderer images, respectively (Gredebäck et al., 2015). One 12-month-old infant met all other inclusion criteria but had only nine artifact-free trials (i.e., one trial short) for hinderer images. Due to the disruption of further data collection caused by COVID-19, we decided to include this participant in our data analysis to meet our pre-registered target. This decision was made prior to statistical analysis, and the inclusion/exclusion of this participant did not change the results of the current study. For the 6month age group, the average number of trials included in the final analyses was 5.17 for helping videos, 5.24 for hindering videos, 20.14 for helper images, and 20.76 for hinderer images. For the 12-month age group, the average number of trials included in the final analyses was 5.31 for helping videos, 5.31 for hindering videos, 21.13 for helper images, and 20.59 for hinderer images. 


\subsection{EEG Recording and Data Pre-Processing}

Infants' neural activity was recorded using a 64-channel HydroCel Geodesic Sensor Net $^{1}$ and amplified by a Net Amps 400 amplifier (Electrical Geodesics, Inc., Eugene, OR). The signals were collected at a sampling rate of $1000 \mathrm{~Hz}$ using EGI's Net Station v5.2 software and referenced to the vertex $(\mathrm{Cz})$. Impedances of all sensors were kept below $50 \mathrm{kOhm}$. EEG signal processing was performed using MATLAB v2020b (MathWorks, Natick, MA), EEGLAB v2020.0 (Delorme \& Makeig, 2004), and ERPLAB v8.10 (Lopez-Calderon \& Luck, 2014).

The pre-processing of raw EEG data followed the Maryland Analysis of Developmental EEG (MADE) pipeline (Debnath et al., 2020). First, raw EEG data, event markers, and channel locations were imported to MATLAB using the MffMatlabIO EEGLAB plugin. The data were then adjusted for anti-aliasing filter and event marker time offsets, and down sampled to $250 \mathrm{~Hz}$. A high-pass filter $(0.3 \mathrm{~Hz})$ and a low-pass filter $(50 \mathrm{~Hz})$ were applied to the data using the FIRfilt EEGLAB plugin. Bad channels (including data with an absolute $\mathrm{Z}$ score greater than 3 ) were detected and removed using the FASTER EEGLAB plugin. Next, a copy/ICA procedure (see Debnath et al., 2020 for details) was performed. Specifically, we created a copy of the original data, applied a high-pass filter $(1 \mathrm{~Hz})$ to the copy, and removed channels and epochs with excessive artifacts from the copy. Independent component analysis (ICA) was then performed on the copy, and ICA weights were transferred back to the original data. Independent Components (ICs) that corresponded to artifacts were identified using the adjusted-ADJUST script (Leach et al., 2020) and removed from the original data. Finally, we interpolated bad channels using spherical spline interpolation, and re-referenced the data to the average of all sensors.

\footnotetext{
${ }^{1}$ EGI Nets made for subjects under the age of 3 do not have built-in eye channels.
} 


\subsection{EEG Data Processing for Familiarization Trials}

After pre-processing, continuous EEG data were segmented for helping and hindering videos from $-2000 \mathrm{~ms}$ prior to the video onset to $17000 \mathrm{~ms}$ after the onset. The data were then visually inspected; trials with less than $70 \%$ looking time and trials with excessive movement artifacts were removed. The helping/hindering videos were divided into phases (see Figure 2), including Baseline (the black screen immediately before the video; -2000-0ms), Pre-phase (video outset to helper/hinderer appearance, includes climber's failed attempts; 0-9500ms), Duringphase (helper/hinderer appearance to end of helping/hindering action; 9500-14000ms), and Postphase (end of helping/hindering action to helper/hinderer's exit; 14000-16800ms).

For frontal alpha asymmetry analysis, the data were segmented into $500 \mathrm{~ms}$ windows with $50 \%$ overlap. For each segment, artifact rejection was performed using a $-200 \mu \mathrm{V}$ to 200 $\mu \mathrm{V}$ threshold. For the remaining segments, power density was computed using a Fast Fourier Transform with a Hanning window, and average power density was calculated across segments and trials for each participant, condition (helping/hindering), and phase (Baseline, Pre-, During-, Post-). For the 6-month age group, the average number of usable segments was 387.14 for helping videos and 393.49 for hindering videos. For the 12-month age group, the average number of usable segments was 401.81 for helping videos and 401.14 for hindering videos.

To compute frontal alpha asymmetry scores, average power density for left (F3/E12) and right (F4/E60) frontal channels in the 5-8 Hz frequency band was natural-log transformed, and difference scores $(\ln [\mathrm{F} 4]-\ln [\mathrm{F} 3])$ were calculated. Due to the inverse relationship between alpha power and brain activation, greater frontal alpha asymmetry scores represent greater alpha power in the right hemisphere and greater activation in the left hemisphere. This calculation approach 
has been widely used in past research examining frontal alpha asymmetry (Buss et al., 2003;

Crespo-Llado et al., 2018; Smith \& Bell, 2010). The frequency band and channels were selected based on Cowell and Decety (2015) and preregistered on OSF. Because frontal alpha asymmetry scores represent relative spectral powers between hemispheres, the scores do not require baseline-correction (see Hwang et al., 2020).

\subsection{EEG Data Processing for Test Trials}

Pre-processed EEG data were segmented from $200 \mathrm{~ms}$ prior to the onset of helper/hinderer image to $1000 \mathrm{~ms}$ after the onset, and baseline corrected using the mean amplitude of the $200 \mathrm{~ms}$ preceding stimulus onset. Artifact detection was performed using a $200 \mu \mathrm{V}$ to $200 \mu \mathrm{V}$ threshold; trials with more than 5 channels exceeding this threshold and trials during which infants looked away from the screen (based on video coding) were rejected. For the remaining trials, channels containing excessive artifacts were replaced using spherical spline interpolation. The signals were then averaged across trials for each condition (helper/hinderer).

Average P400 amplitude was extracted from the $250-400 \mathrm{~ms}$ time window over channels in the occipitotemporal area (left hemisphere: E31, E33, right hemisphere: E40, E38). Average $\mathrm{Nc}$ amplitude was extracted from the $400-600 \mathrm{~ms}$ time window over channels in the frontocentral area (E20, E15, E16, E7, E4, E54, E51, E53, E50; see Figure 3). The component time windows and channels were chosen based on Gredebäck et al. (2015) and confirmed through visual inspection of grand-average waveforms (Kappenman \& Luck, 2016).

As an exploratory analysis, we computed the average amplitude of the N290 component as a supplementary measure of socially relevant processes. The N290 is thought to be topographically and functionally related to the $\mathrm{P} 400$ component, and is sensitive to social stimuli 
such as faces (for review, see Csibra et al., 2008; de Haan et al., 2003). Note that this measure was not preregistered, but the decision to examine N290 was made prior to analysis. Average N290 amplitude was extracted from the $150-300 \mathrm{~ms}$ time window, using the same channels as the P400 component. The component time window was chosen based on past research (Halit et al., 2003; Leppänen et al., 2007; Luyster et al., 2014) as well as visual inspection of grandaverage waveforms.

\subsection{Analysis Plan}

Statistical analyses were conducted to explore 1) whether frontal alpha asymmetry scores differed between helping and hindering videos, 2) whether infants showed differential ERP (P400, N290, Nc) responses to helper and hinderer images, 3) whether infants showed a grouplevel preference for the helper (vs. hinderer) during manual choice, and 4) whether infants' helper preferences were correlated with frontal alpha asymmetry and ERPs. For all analyses, extreme outliers were detected using the identify_outliers function of the rstatix (version 0.6.0) R package. Values that were 3 interquartile ranges (IQRs) above the upper quartile ( $75^{\text {th }}$ percentile) or below the lower quartile $\left(25^{\text {th }}\right.$ percentile) were marked as extreme outliers and excluded from analysis. As a result, one outlier was removed from frontal alpha asymmetry and N290 analyses (respectively), and two outliers were removed from P400 and Nc analyses (respectively). For analyses of variance (ANOVAs), normality was tested through visual inspection of Q-Q plots of standardized residuals, and violations of sphericity were corrected using the Greenhouse-Geisser method. 
636

(NAS)

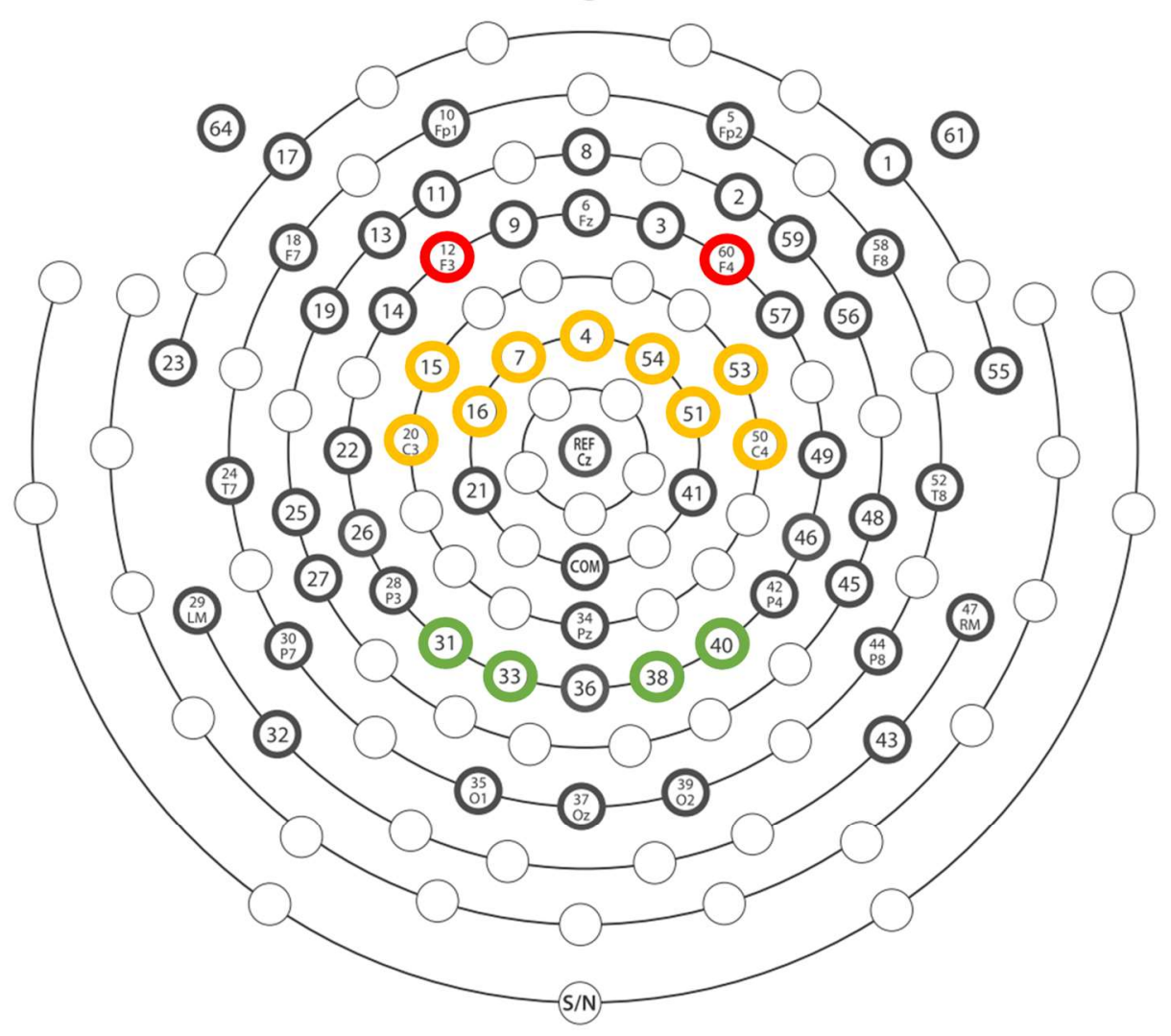

Frontal alpha asymmetry channels:

P400/N290 channels:

:

Nc channels:

Figure 3. Channels used for frontal alpha asymmetry, P400/N290, and Nc analyses. 


\section{Results}

\subsection{Familiarization Trials}

To investigate whether infants' frontal alpha asymmetry scores differed between helping and hindering videos, we conducted a mixed ANOVA with condition (helping, hindering) and phase (Baseline, Pre, During, Post) as within-subjects factors, and age group (6-month, 12month) as a between-subjects factor. Results showed a significant interaction between condition and age group, $F(1,71)=4.08, p=.047, \eta_{p}{ }^{2}=.054$. Post-hoc tests revealed that 6 -month-olds' frontal asymmetry scores (indexing approach motivation) were higher for the helping condition $(M=0.02,95 \% \mathrm{CI}[-0.080 .11])$ than the hindering condition $(M=-0.03,95 \% \mathrm{CI}[-0.130 .07])$, $t(71)=2.18, p=.03$, consistent with our hypothesis and with Cowell \& Decety (2015). No condition differences were found for 12-month-olds, $t(71)=-.77, p=.44, M_{\text {helping }}=0.01, M$ hindering $=0.03$. These results suggest that 6 -month-olds (but not 12-month-olds) showed more left frontal activation (indexing approach motivation) when viewing helping (vs. hindering) events (see also SM 3.1 for supplementary analysis showing that this alpha asymmetry effect was specific to frontal channels).

To explore whether condition differences were more pronounced during specific phases of helping and hindering events, we compared frontal alpha asymmetry between conditions in each phase using paired samples t-tests. When Shapiro-Wilk tests showed violations of normality, we performed Wilcoxon signed-ranks tests instead. As shown in Figure 4, 6-montholds showed significantly higher frontal alpha asymmetry for helping (vs. hindering) in the During-phase (as the helping/hindering actions took place), $M_{\text {helping }}=0.07, M_{\text {hindering }}=-0.03$, $t(41)=2.45, p=.019,95 \%$ CI [0.02 0.18], but not in other phases (e.g., before/after 
helping/hindering), $p \mathrm{~s}>.05$. No condition differences were found in any phases for 12-montholds, $p \mathrm{~s}>.05$. These results suggest that 6-month-olds showed more approach motivation when helping (vs. hindering) actions took place. We will return to this issue in the discussion.

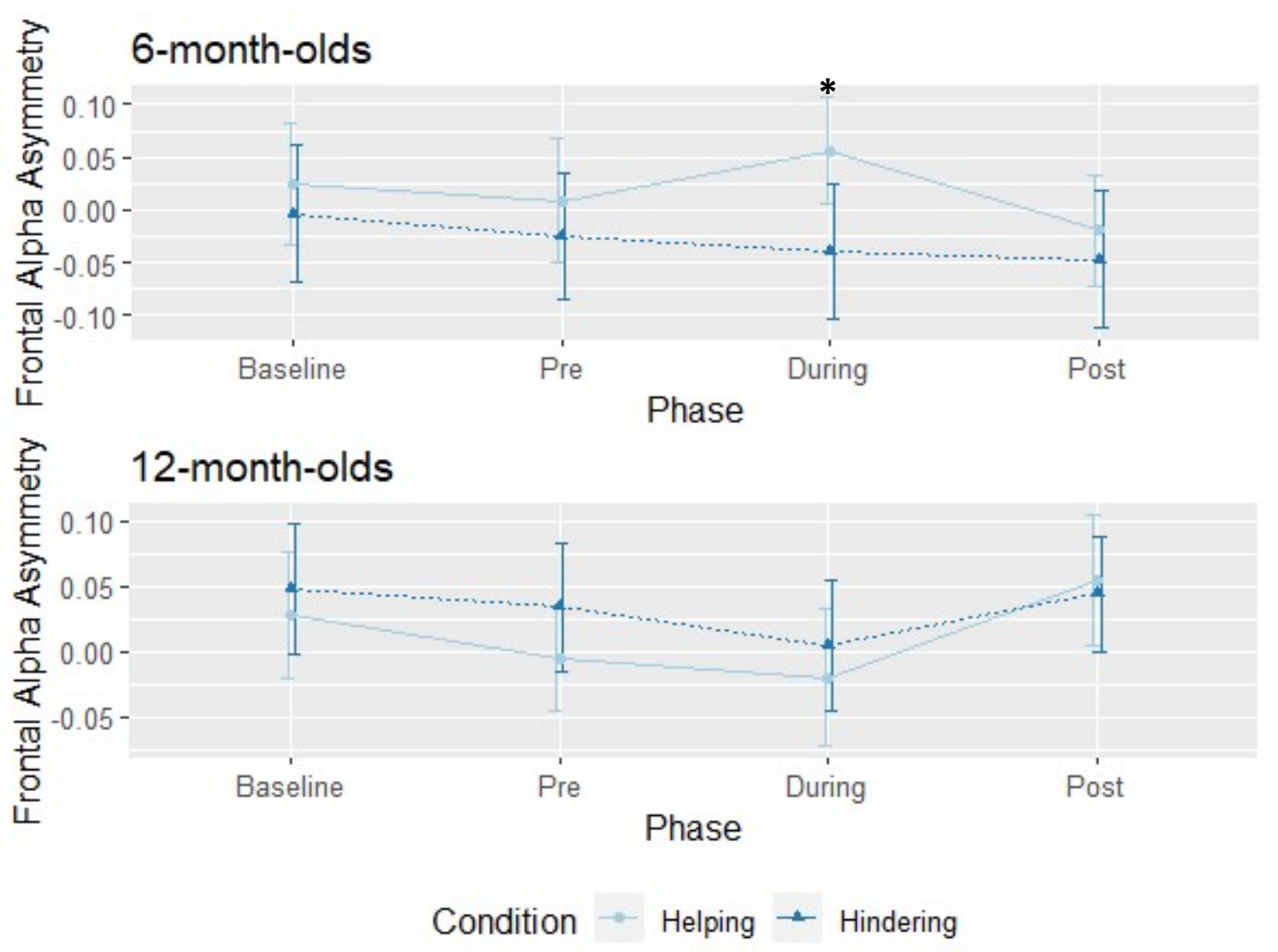

Figure 4. Frontal alpha asymmetry scores (right hemisphere - left hemisphere) during each phase. Greater scores are associated with higher approach (vs. avoidance) motivation. Asterisks represent significant differences between helping and hindering events at $p<.05$. Error bars represent standard errors.

\subsection{Test Trials}

To investigate whether infants showed differential P400 responses to helper and hinderer images, we entered P400 mean amplitudes into a 2 (condition: helping, hindering) $\times 2$ 
(hemisphere: left, right) $\times 2$ (age group: 6-month, 12-month) mixed ANOVA. Results found a main effect of condition, $F(1,70)=4.83, p=.031, \eta^{2}=.07$ (see Figure 5), a main effect of age, $F(1$, $70)=13.70, p<.001, \eta^{2}=.16$, and a main effect of hemisphere $F(1,70)=10.26, p=.002, \eta^{2}=.13$. No significant interactions were found, $p \mathrm{~s}>.05$. Specifically, $\mathrm{P} 400$ mean amplitude was higher for hinderer $(M=5.40,95 \% \mathrm{CI}[3.53$ 7.27]) than helper $(M=3.27,95 \% \mathrm{CI}[1.405 .13])$ images, higher for 12-month-olds $(M=7.35,95 \% \mathrm{CI}[5.06$ 9.64]) than 6-month-olds ( $M=1.32,95 \% \mathrm{CI}[-0.97$ 3.61]), and higher for the right hemisphere ( $M=5.46,95 \%$ CI [3.71 7.21]) than the left hemisphere ( $M=3.21,95 \%$ CI [1.46 4.96]). Supplementary analyses using an alternative time window for 12-month-olds (SM 3.2) and including number of images viewed as a covariate (SM 3.3) found similar results. These results provide evidence that images of helper and hinderer elicited differential P400 responses in infants, and that this effect was consistent across age groups. Considering that the $\mathrm{P} 400$ component is thought to be an index of social processing (Gredebäck \& Daum, 2015; Gredebäck et al., 2015), these findings suggest that socially relevant neural processes are involved when infants view prosocial versus antisocial characters. Further, higher P400 amplitudes for hinderer (vs. helper) are consistent with past research showing that infants might be more sensitive to socially relevant information in entities causing negative (vs. positive) outcomes (Hamlin \& Baron, 2014; see discussion below).

For the Nc component, a 2 (condition: helping, hindering) $\times 2$ (age group: 6-month, 12month) mixed ANOVA found higher Nc mean amplitude for 6-month-olds ( $M=-1.30,95 \% \mathrm{CI}[-$ $3.160 .56])$ than 12 -month-olds $(M=-8.41,95 \%$ CI $[-10.27-6.55]), F(1,70)=28.90, p<.001$, $\eta^{2}=.29$. No main effect or interactions were found for condition, $p \mathrm{~s}>.05$ (see Figure 5). Similar results were found when the number of images viewed was included as a covariate (SM 3.3). These results demonstrate that infants did not show differential Nc responses (indexing 
attentional allocation) when viewing helper versus hinderer images, suggesting that the condition differences observed in the $\mathrm{P} 400$ component were not attributable to general attentional differences.

For the N290 component, an exploratory 2 (condition: helping, hindering) $\times 2$ (hemisphere: left, right) $\times 2$ (age group: 6-month, 12-month) mixed ANOVA found a significant main effect of age group, $F(1,71)=7.17, p=.009, \eta^{2}=.09$, and a marginally significant main effect of condition, $F(1,71)=3.99, p=.050, \eta^{2}=.05$ (see Figure 5). Specifically, N290 mean amplitude was higher for hinderer $(M=1.34,95 \% \mathrm{CI}[-0.353 .03])$ than helper $(M=-0.49,95 \% \mathrm{CI}$ [-2.18 1.21]), and higher for 12-month-olds ( $M=2.38,95 \%$ CI [0.34 4.43]) than 6-month-olds $(M=-1.52,95 \%$ CI $[-3.570 .52])$. Similar results were found when the number of images viewed was included as a covariate (SM 3.3). These results provide further evidence that infants showed differential responses in the N290/P400 complex when viewing images of the helper versus hinderer, supporting the idea that infants' responses to prosocial versus antisocial characters involve socially relevant processes. 

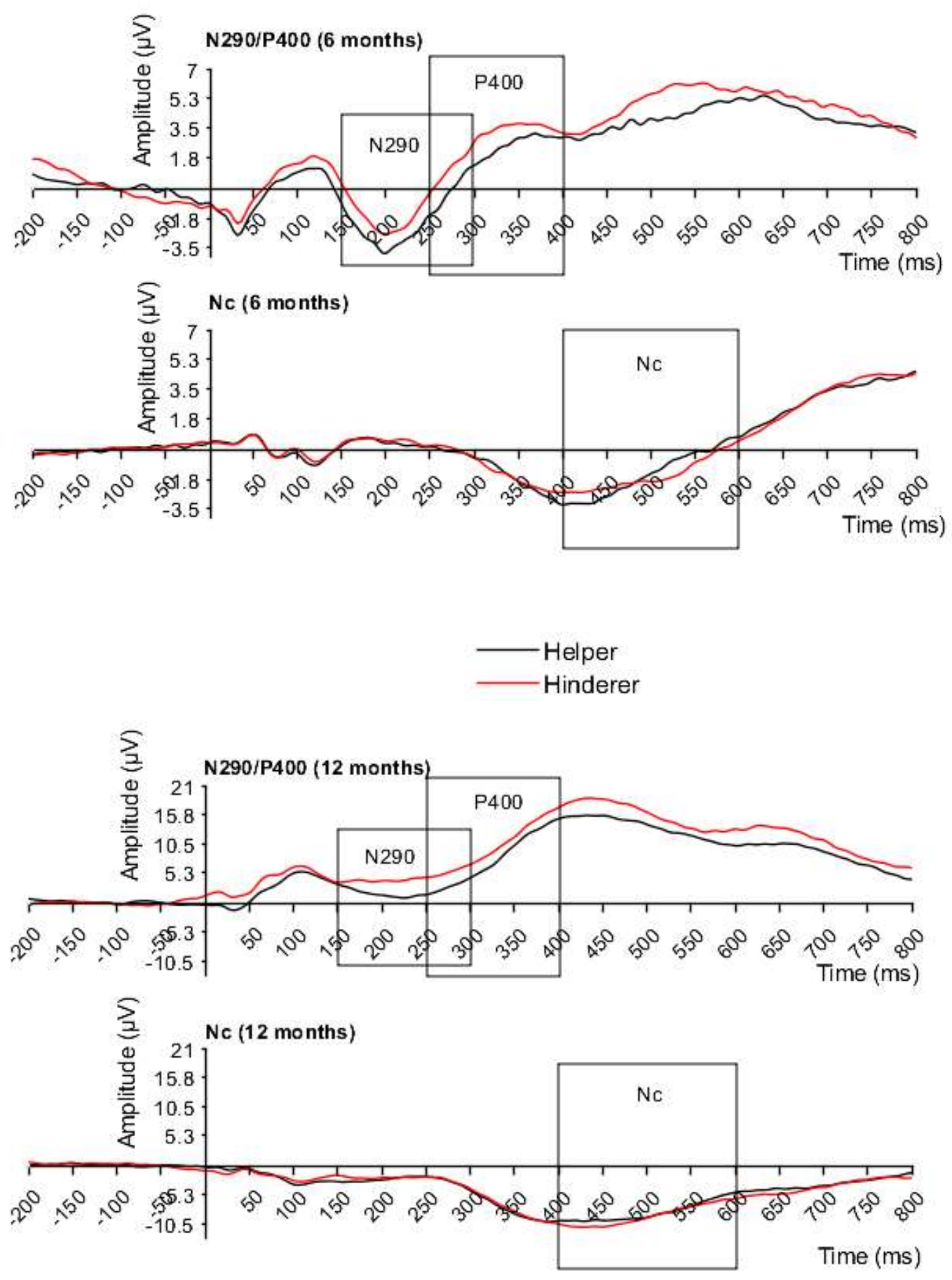

Figure 5. Grand average waveforms for the first $800 \mathrm{~ms}$ after the onset of helper/hinderer images, showing the P400, N290, and Nc components over their corresponding channels. 


\subsection{Manual Choice}

To investigate whether infants showed a group-level preference for the helper, we examined infants' manual choice between the helper and the hinderer using binomial tests. For 6-month-olds, 15 out of the 38 infants who made a choice (4 infants did not choose) preferred the helper over the hinderer (binomial test, $p=.256$ ). For 12-month-olds, 11 out of the 24 infants who made a choice ( 8 infants did not choose) preferred the helper over the hinderer (binomial test, $p=.839)$. The percentage of infants choosing the helper over the hinderer did not differ between age groups, $\chi^{2}(1, \mathrm{~N}=62)=0.05, p=.818$. These results demonstrate that neither 6-month-olds nor 12-month-olds in the current study showed a group-level manual preference for the helper.

\subsection{Correlations Between Behavioral and EEG Measures}

To explore the relations between infants' manual preferences and EEG responses, we computed condition difference scores (helping - hindering) for frontal alpha asymmetry in the During- as well as the Post-phases of familiarization trials, and for P400, N290, and Nc mean amplitudes during test trials. We then correlated these condition difference scores with infants' tendency to choose the helper during manual choice. Because visual inspection and ShapiroWilk's tests showed violations of normality in several EEG measures, we report Spearman correlation coefficients.

For 6-month-olds, analyses found no significant correlations between EEG measures and infants' manual preferences for the helper, $p \mathrm{~s}>.05$. For 12-month-olds, infants' helper preferences were correlated with differential frontal alpha asymmetry scores in the Post-phase, after helping/hindering took place. Specifically, infants who showed higher relative right frontal alpha power (indexing approach motivation) after helping (vs. hindering) were less likely to 
choose the helper, $r_{s}(22)=-.59, p=.003$ (this effect survived a Bonferroni correction: $\alpha_{\text {adjusted }}$ $=.05 / 12=.004)$. Follow-up analyses revealed that this effect was mainly driven by the helping condition. Within the helping scenario, infants who showed higher right frontal alpha power during the Post-phase were (somewhat) less likely to choose the helper, $r_{s}(22)=-.40, p=.050$ (this effect did not survive a Bonferroni correction, $p>$.004). No significant effects were found for the hindering scenario, $p=.593$. These results suggest that infants' manual preferences might be related to their approach-avoidance motivation during the Post-phase of the scenario. That said, we note that we did not find group-level asymmetry effects in 12-month-olds, that these results were based on a relatively small sample of infants who made a choice $(\mathrm{N}=24)$, and that infants were mostly quite fussy during the manual preference procedure (see discussion below). Hence, these effects should be interpreted with caution.

\section{Discussion}

The current study used EEG to explore infants' neural responses to prosocial and antisocial interactions and characters. We found that 6-month-old (but not 12-month-old) infants showed higher relative right frontal alpha power (indexing approach motivation) when viewing helping versus hindering. These findings provide evidence that motivational processes are implicated when infants process prosocial/antisocial interactions, and that observing helping (vs. hindering) actions elicits more approach (vs. avoidance) tendencies in 6-month-olds, but not 12month-olds. Infants did not show a group-level manual preference for the helper. However, analyses of infants' ERP responses to still images of the helper and hinderer revealed that both 6and 12-month-olds showed differential ERP responses to images of the helper and hinderer in the P400 and N290 (indexing social processing) but not in the Nc (indexing attentional allocation). We note that the direction of the P400 effect was in the opposite direction of that found in 
Gredebäck et al. (2015). That said, these ERP results are consistent with the hypothesis that infants' reactions to prosocial and antisocial others are supported by socially relevant neural mechanisms.

Our finding that 6-month-olds showed greater right frontal alpha power during helping (vs. hindering) scenarios is consistent with Cowell and Decety (2015); however, we did not replicate the same effect in 12-month-olds (see discussion below). Together, these results highlight the importance of frontal brain activity in infants' responses as sociomoral events unfold. Relatedly, a recent study found that greater resting state right frontal alpha power at 14 months predicted better understanding of others' distress and empathic responding at 24 months (Paulus et al., 2013), providing evidence for the links between frontal brain activity and the development of early prosocial tendencies. We note that the high temporal resolution of EEG and the use of phase-specific analysis in the current study allowed us to more precisely pinpoint when differences in infants' neural responses emerged between conditions. Notably, significant condition differences (for 6-month-olds) emerged in the During-phase, as the helping/hindering occurred, but not in any other phases. This temporal specificity suggests that infants' frontal alpha asymmetry responses were specifically evoked by prosocial/antisocial acts per se, as opposed to other aspects of the displays; for example the positive versus negative outcomes caused by those acts.

How should this frontal asymmetry effect be interpreted? Past research has shown that greater right frontal alpha in infants is associated with approach behaviors (e.g., hand reaching and positive vocalization during approach of mother), and that greater left frontal alpha is associated with avoidance/withdrawal behaviors (e.g., gaze aversion and distress during approach of stranger) (Fox \& Davidson, 1987; Fox \& Davidson, 1988). The results of the current 
study suggest that 6-month-olds exhibit more approach tendencies as they observe prosocial (vs. antisocial) interactions unfolding. One possibility is that these approach-avoidance tendencies represent precursors of sociomoral evaluations. Specifically, infants' frontal alpha asymmetry may represent intuitive, automatic approach/avoidance responses, that then feed into a decisionmaking system that generates positive/negative sociomoral evaluations and subsequent behavioral approach tendencies. This interpretation is in line with the social intuitionist model of moral judgments in the adult literature, which posits that moral decisions derive from automatic/intuitive response to sociomoral scenes, but that deliberative processes may be recruited after the fact to provide rationalization/justification (Haidt, 2001, 2007). Alternatively, infants' frontal alpha asymmetry could represent some outcome of (rather than precursors to) sociomoral evaluations; that is, it could reflect that some evaluation has already occurred. We note that the asymmetry effect observed was specific to the During-phase as helping/hindering occurred, suggesting that the first possibility is more likely to account for the observed alpha asymmetry. However, supplementary analyses (see SM 3.4) revealed that infants' frontal alpha asymmetry during helping/hindering events was unrelated to their P400, N290, and Nc responses to still images of the helper and hinderer. These findings suggest that infants' approachavoidance responses during helping/hindering events were relatively independent of their neural responses to helper/hinderer images, offering support for the second possibility. Future studies should clarify the precise role of frontal alpha responses in sociomoral evaluations, by systematically examining their time course across distinct sociomoral contexts.

Cowell and Decety (2015) found a frontal alpha asymmetry effect in a relatively older sample (12- to 24 -month-olds; mean age $=18.69$ months), whereas in the current study we found the effect in 6-month-olds but not in 12-month-olds. Although this discrepancy might be 
attributable to statistical power differences due to sample size differences ( $\mathrm{N}=42$ for 6-montholds and N=32 for 12-month-olds), visual inspection of descriptive statistics (see Figure 4) suggests that 12-month-olds in the current study showed a different data pattern from 6-montholds. One interpretation of this age difference is that compared with 12-month-olds, 6-montholds are more sensitive to prosocial and antisocial interactions and so exhibit stronger frontal alpha asymmetry effects. Consistent with this idea, a meta-analysis found that the correlations between infant resting state frontal alpha asymmetry and maternal depression were stronger in younger (vs. older) infants (Thibodeau et al., 2006), providing evidence for age differences in frontal alpha asymmetry responses. Alternatively, 12-month-olds may process helper versus hinderer events in some other way. Future studies should further explore whether younger infants show stronger frontal asymmetry responses to prosocial/antisocial interactions, and whether and how infants' neural responses to prosocial and antisocial interactions change over time.

The second main goal of the current study was to explore whether infants' responses to prosocial and antisocial characters are social in nature. To this end, we examined whether images of the helper and the hinderer elicited differential responses in neural signatures of socially relevant processes (P400/N290) or in non-social, domain-general processes $(\mathrm{Nc})$. Results found that, across two age groups, infants showed differential ERP responses in the P400 and N290 (the latter was marginally significant), but not in the Nc. The fact that we found similar patterns of data across two distinct age groups (6- and 12-month-olds) demonstrates the robustness of these effects. This developmental continuity is consistent with behavioral findings showing that infants' social preferences for prosocial over antisocial characters are stable between 4 and 32 months of age (Margoni \& Surian, 2018). Taken together, these results support the hypothesis 
that that infants' responses to prosocial and antisocial characters are based on socially relevant processes.

The P400 effect found in the current study was in the opposite direction of that observed in Gredebäck et al. (2015). Specifically, Gredebäck and colleagues (2015) found larger P400 amplitudes when infants viewed images of the helper versus the hinderer, whereas here we found that infants showed larger P400 amplitudes for the hinderer versus the helper. We note that the current study found consistent P400 (and N290) effects across two age groups and with considerably larger sample sizes (total N=74) than Gredebäck et al. (2015) (total N=14). Hence, our results may represent a more reliable estimate of infants' P400 responses to prosocial/antisocial scenarios. Furthermore, we view our results as consistent with a larger body of ERP and behavioral findings. For instance, past behavioral research has shown that infants may attribute agency and goal-directedness to inanimate entities that cause negative, but not that cause positive, outcomes (Hamlin \& Baron, 2014), suggesting that infants might also attribute relatively more agency to animate hinderers versus helpers. As past ERP studies have found larger P400 amplitudes when infants observe agents engaging in functional (vs. non-functional), congruent (vs. incongruent), and goal-directed (vs. non-goal directed) actions (Bakker et al., 2015; Bakker et al., 2016; Gredebäck et al., 2010; but see Melinder et al., 2015), it may be the P400 component reliably differentiates the amount of agency attributed to a given agent or act. Further, our results are also consistent with research showing that negative (vs. positive) facial expressions and untrustworthy (vs. trustworthy) faces elicit larger P400 responses in infants (Jessen \& Grossmann, 2015, 2016; Leppänen et al., 2007), suggestive that the P400 may distinguish negative versus positive social stimuli more broadly. Further study of infants' P400 
responses to prosocial/antisocial characters in additional contexts will help to better clarify the nature of these effects.

Although both the current study and Gredebäck et al. (2015) found no Nc responses to helper/hinderer images, past research suggests that the Nc might be sensitive to prosocial/antisocial interactions. For instance, using a paradigm different from the hill scenario, Cowell and Decety (2015) found that infants showed differential Nc responses when viewing pictures depicting various types of prosocial/antisocial interactions. Further, infants' Nc responses were associated with their helper preferences in the hill paradigm, as well as their parents' sensitivity to injustice. Taken together, these findings suggest that the $\mathrm{Nc}$ component (indexing attentional processes) could be implicated in infants' processing of prosocial/antisocial interactions, whereas the P400 and N290 components might be more involved in infants' evaluations of prosocial/antisocial characters. Unfortunately, the design of the current study did not allow us to test this hypothesis directly, because the helping/hindering interactions were enacted by videos (as opposed to pictures) and hence infants' $\mathrm{Nc}$ responses (e.g., to helping/hindering actions) would necessarily be contaminated by continuous perceptual input from the videos. To test this hypothesis, future studies should examine infants' P400/N290 and Nc responses to sociomoral interactions depicted by static images.

The current study did not find a group-level helper preference in either age group. These results are consistent with some recent findings using the hill paradigm (Cowell \& Decety, 2015; Schlingloff et al., 2020; but see positive evidence in Hamlin, 2015; Loheide-Niesmann et al., 2021; Tan \& Hamlin, 2021). While acknowledging the inconsistencies in infants' helper preferences to date, we view infants' failure in the current studies as relatively unsurprising. Indeed, in the current study infants wore EEG caps (which may undermine behavioral 
performance; see Cowell \& Decety, 2015; Filippi et al., 2016), were only familiarized (vs. habituated) to the helping/hindering interactions, and viewed cartoon as opposed to live 3-D stimuli. Perhaps most importantly, the manual choice task in the current study did not start until after infants had willfully stopped attending to the stimuli, as obtaining sufficient trials for EEG/ERP analyses was our primary goal. That is, by the time infants entered the choice task they were already quite inattentive and fussy; many infants failed to choose between the characters at all. Therefore, and given recent evidence that even younger, 5-month-old infants show visual preferences for the helper after sufficient exposure to the very same cartoon hill stimuli in a behavioral paradigm (Tan \& Hamlin, 2021), we suspect that the manual choice measure in the current study was not a particularly reliable or valid measure of infants' social preferences.

With respect to correlational analyses, we found that infants who showed higher right frontal alpha power during the Post-phase to helping (vs. hindering) events were less likely to choose the helper, suggesting that infants who showed more approach tendencies when the helper left the scene actually preferred the helper less; this effect was against our prediction. That said, the correlational results should be interpreted with caution, in light of the previous discussion of the (likely) low reliability of the choice measure; evidence for or against this relationship should be confirmed in future studies in which infants are less fatigued during choice. Future research should also incorporate other infant-friendly techniques to explore how social preferences are linked to activation in specific brain areas. For example, using functional near-infrared spectroscopy (fNIRS) and eye-tracking, recent studies have shown that neural activity in the medial prefrontal cortex (mPFC) is associated with infants' (visual) preferences for those who previously smiled versus frowned at them, and that genetic variation in the 
oxytocin system is linked to individual differences in these effects (Krol \& Grossmann, 2020; Krol et al., 2021).

\section{Conclusions}

The current study provides new evidence that approach/avoidance mechanisms are involved in 6-month-old infants' processing of helping and hindering scenarios, and that, for both 6- and 12-month-olds, images of the helper and hinderer elicit neural responses associated with social processing (P400, N290) but not those associated with attentional allocation (Nc). Together, these findings add to a growing literature showing that sociomoral evaluations are based on an intricate interplay between various cognitive, social, emotional, and motivational mechanisms (Cowell et al., 2018; Young \& Dungan, 2012). We note that some of the effects found in the current study were different from those observed in prior research (Cowell \& Decety, 2015; Gredebäck et al., 2015; Hamlin et al., 2007), including the lack of alpha frontal asymmetry in 12-month-olds, the lack of a group-level manual preference for the helper, and the reversed P400 effects in both age groups. Hence, more research is needed to clarify the robustness and direction of these effects. Future studies should also explore what specific purposes these neural mechanisms serve in infants' responses to sociomoral scenarios, and whether the role of these mechanisms in sociomoral processing changes as infants age. 
Acknowledgments: The authors have no conflict of interest to declare. We thank Hannah de Vries, Vivian Wong, Rachelle Graham, Fibha Khan, Julia Van de Vondervoort, Francis Yuen, Julia Mermier, Emily Mah, and Evangeline Wong for their assistance. Special thanks go to Brandon M. Woo for creating the stimuli.

Funding: This work was supported in part by the Natural Sciences and Engineering Research Council of Canada [NSERC; grant number RGPIN-2016-03775] to J. Kiley Hamlin. The funding source was not involved in study design, analysis of data, interpretation of results, or writing of the report.

Data availability statement: All data are available from the corresponding author upon reasonable request.

Declaration of competing interest: The authors declare that they have no known competing financial interests or personal relationships that could have appeared to influence the work reported in this paper. 


\section{References}

Bakker, M., Daum, M. M., Handl, A., \& Gredebäck, G. (2015). Neural correlates of action perception at the onset of functional grasping. Social cognitive and affective neuroscience, 10(6), 769-776.

Bakker, M., Sommerville, J. A., \& Gredebäck, G. (2016). Enhanced neural processing of goaldirected actions after active training in 4-month-old infants. Journal of Cognitive Neuroscience, 28(3), 472-482.

Buon, M., Jacob, P., Margules, S., Brunet, I., Dutat, M., Cabrol, D., \& Dupoux, E. (2014). Friend or foe? Early social evaluation of human interactions. PLoS One, 9(2), e88612.

Buss, K. A., Schumacher, J. R. M., Dolski, I., Kalin, N. H., Goldsmith, H. H., \& Davidson, R. J. (2003). Right frontal brain activity, cortisol, and withdrawal behavior in 6-month-old infants. Behavioral Neuroscience, 117(1), 11-20.

Cowell, J. M., Calma-Birling, D., \& Decety, J. (2018). Domain-general neural computations underlying prosociality during infancy and early childhood. Current Opinion in Psychology, 20, 66-71. https://doi.org/10.1016/j.copsyc.2017.08.016

Cowell, J. M., \& Decety, J. (2015). Precursors to morality in development as a complex interplay between neural, socioenvironmental, and behavioral facets. Proceedings of the National Academy of Sciences, 112(41), 12657-12662. https://doi.org/10.1073/pnas.1508832112

Crespo-Llado, M. M., Vanderwert, R., Roberti, E., \& Geangu, E. (2018). Eight-month-old infants' behavioral responses to peers' emotions as related to the asymmetric frontal cortex activity. Scientific reports, 8(1), 1-11. https://doi.org/10.1038/s41598-018-35219-4 
Csibra, G., Kushnerenko, E., \& Grossmann, T. (2008). Electrophysiological methods in studying infant cognitive development. In C. A. Nelson \& M. Luciana (Eds.), Handbook of Developmental Cognitive Neuroscience (second ed.). MIT Press.

de Haan, M., Johnson, M. H., \& Halit, H. (2003). Development of face-sensitive event-related potentials during infancy: a review. International Journal of Psychophysiology, 51(1), $45-58$.

Debnath, R., Buzzell, G. A., Morales, S., Bowers, M. E., Leach, S. C., \& Fox, N. A. (2020). The Maryland analysis of developmental EEG (MADE) pipeline. Psychophysiology, 57(6), e13580. https://doi.org/10.1111/psyp.13580

Delorme, A., \& Makeig, S. (2004). EEGLAB: an open source toolbox for analysis of single-trial EEG dynamics including independent component analysis. Journal of Neuroscience Methods, 134(1), 9-21. https://doi.org/10.1016/j.jneumeth.2003.10.009

Filippi, C. A., Cannon, E. N., Fox, N. A., Thorpe, S. G., Ferrari, P. F., \& Woodward, A. L. (2016). Motor System Activation Predicts Goal Imitation in 7-Month-Old Infants. Psychological Science, 27(5), 675-684. https://doi.org/10.1177/0956797616632231

Fox, N., \& Davidson, R. (1987). EEG asymmetry in ten month old infants in response to approach of a stranger and maternal separation. Developmental psychology, 23, 233-240.

Fox, N. A., \& Davidson, R. J. (1988). Patterns of brain electrical activity during facial signs of emotion in 10-month-old infants. Developmental psychology, 24(2), 230-236.

Geraci, A., \& Surian, L. (2011). The developmental roots of fairness: Infants' reactions to equal and unequal distributions of resources. Developmental Science, 14(5), 1012-1020. 
Gredebäck, G., \& Daum, M. M. (2015). The microstructure of action perception in infancy: Decomposing the temporal structure of social information processing. Child Development Perspectives, 9(2), 79-83.

Gredebäck, G., Kaduk, K., Bakker, M., Gottwald, J., Ekberg, T., Elsner, C., . . Kenward, B. (2015). The neuropsychology of infants' pro-social preferences. Developmental cognitive neuroscience, 12, 106-113.

Gredebäck, G., Melinder, A., \& Daum, M. (2010). The development and neural basis of pointing comprehension. Social neuroscience, 5(5-6), 441-450.

Haidt, J. (2001). The emotional dog and its rational tail: a social intuitionist approach to moral judgment. Psychological review, 108(4), 814-834.

Haidt, J. (2007). The new synthesis in moral psychology. science, 316(5827), 998-1002.

Halit, H., De Haan, M., \& Johnson, M. (2003). Cortical specialisation for face processing: facesensitive event-related potential components in 3-and 12-month-old infants. Neuroimage, 19(3), 1180-1193.

Hamlin, J. K. (2013). Failed attempts to help and harm: Intention versus outcome in preverbal infants' social evaluations. Cognition, 128(3), 451-474. https://doi.org/10.1016/j.cognition.2013.04.004

Hamlin, J. K. (2015). The case for social evaluation in preverbal infants: Gazing toward one's goal drives infants' preferences for Helpers over Hinderers in the hill paradigm. Frontiers in psychology, 5, 1563. https://doi.org/10.3389/fpsyg.2014.01563

Hamlin, J. K., \& Baron, A. S. (2014). Agency attribution in infancy: Evidence for a negativity bias. PLoS One, 9(5), e96112. 
Hamlin, J. K., \& Wynn, K. (2011). Young infants prefer prosocial to antisocial others. Cognitive Development, 26(1), 30-39. https://doi.org/10.1016/j.cogdev.2010.09.001

Hamlin, J. K., Wynn, K., \& Bloom, P. (2007). Social evaluation by preverbal infants. Nature, 450(7169), 557-559. https://doi.org/10.1038/nature06288

Hamlin, J. K., Wynn, K., \& Bloom, P. (2010). Three-month-olds show a negativity bias in their social evaluations. Developmental Science, 13(6), 923-929. https://doi.org/10.1111/j.1467-7687.2010.00951.x

Harmon-Jones, E., \& Gable, P. A. (2018). On the role of asymmetric frontal cortical activity in approach and withdrawal motivation: An updated review of the evidence. Psychophysiology, 55(1), e12879. https://doi.org/10.1111/psyp.12879

Holvoet, C., Scola, C., Arciszewski, T., \& Picard, D. (2016). Infants' preference for prosocial behaviors: A literature review. Infant Behavior and Development, 45, Part B, 125-139. https://doi.org/10.1016/j.infbeh.2016.10.008

Hrdy, S. B. (1999). Mother Nature: a history of mothers, infants and natural selection. Pantheon Press.

Hwang, H. G., Debnath, R., Meyer, M., Salo, V. C., Fox, N. A., \& Woodward, A. (2020). Neighborhood racial demographics predict infants' neural responses to people of different races. Developmental Science, e13070.

Jessen, S., \& Grossmann, T. (2015). Neural signatures of conscious and unconscious emotional face processing in human infants. Cortex, 64, 260-270.

Jessen, S., \& Grossmann, T. (2016). Neural and behavioral evidence for infants' sensitivity to the trustworthiness of faces. Journal of Cognitive Neuroscience, 28(11), 1728-1736.

Joyce, R. (2006). The evolution of morality. The MIT Press. 
Kappenman, E. S., \& Luck, S. J. (2016). Best Practices for Event-Related Potential Research in Clinical Populations. Biological Psychiatry: Cognitive Neuroscience and Neuroimaging, 1(2), 110-115. https://doi.org/10.1016/j.bpsc.2015.11.007

Krol, K. M., \& Grossmann, T. (2020). Impression formation in the human infant brain. Cerebral cortex communications, $1(1)$, tgaa070.

Krol, K. M., Namaky, N., Monakhov, M. V., Lai, P. S., Ebstein, R., \& Grossmann, T. (2021). Genetic variation in the oxytocin system and its link to social motivation in human infants. Psychoneuroendocrinology, 131, 105290. https://doi.org/https://doi.org/10.1016/j.psyneuen.2021.105290

Leach, S. C., Morales, S., Bowers, M. E., Buzzell, G. A., Debnath, R., Beall, D., \& Fox, N. A. (2020). Adjusting ADJUST: Optimizing the ADJUST algorithm for pediatric data using geodesic nets. Psychophysiology, 57(8), e13566.

Leppänen, J. M., Moulson, M. C., Vogel-Farley, V. K., \& Nelson, C. A. (2007). An ERP study of emotional face processing in the adult and infant brain. Child development, 78(1), 232245.

Loheide-Niesmann, L., de Lijster, J., Hall, R., van Bakel, H., \& Cima, M. (2021). Toddlers' preference for prosocial versus antisocial agents: No associations with empathy or attachment security. Social Development, 30(2), 410-427. https://doi.org/https://doi.org/10.1111/sode.12487

Lopez-Calderon, J., \& Luck, S. J. (2014). ERPLAB: an open-source toolbox for the analysis of event-related potentials [Technology Report]. Frontiers in Human Neuroscience, 8(213). https://doi.org/10.3389/fnhum.2014.00213 
Luyster, R. J., Powell, C., Tager-Flusberg, H., \& Nelson, C. A. (2014). Neural measures of social attention across the first years of life: Characterizing typical development and markers of autism risk. Developmental cognitive neuroscience, 8, 131-143. https://doi.org/10.1016/j.den.2013.09.006

Margoni, F., \& Surian, L. (2018). Infants' evaluation of prosocial and antisocial agents: A metaanalysis. Developmental psychology, 54(8), 1445-1455. https://doi.org/10.1037/dev0000538

Melinder, A. M., Konijnenberg, C., Hermansen, T., Daum, M. M., \& Gredebäck, G. (2015). The developmental trajectory of pointing perception in the first year of life. Experimental Brain Research, 233(2), 641-647.

Nelson, C. A., \& Monk, C. S. (2001). The use of event-related potentials in the study of cognitive development. In C. A. Nelson \& M. Luciana (Eds.), Handbook of developmental cognitive neuroscience (pp. 125-136). MIT Press.

Paulus, M., Kühn-Popp, N., Licata, M., Sodian, B., \& Meinhardt, J. (2013). Neural correlates of prosocial behavior in infancy: Different neurophysiological mechanisms support the emergence of helping and comforting. Neuroimage, 66, 522-530.

Reznik, S. J., \& Allen, J. J. (2018). Frontal asymmetry as a mediator and moderator of emotion: An updated review. Psychophysiology, 55(1), e12965.

Scarf, D., Imuta, K., Colombo, M., \& Hayne, H. (2012). Social evaluation or simple association? Simple associations may explain moral reasoning in infants. PLoS One, 7(8), e42698. https://doi.org/10.1371/journal.pone.0042698 
Schlingloff, L., Csibra, G., \& Tatone, D. (2020). Do 15-month-old infants prefer helpers? A replication of Hamlin et al . (2007). Royal Society Open Science, 7(4), 191795. https://doi.org/10.1098/rsos.191795

Scola, C., Holvoet, C., Arciszewski, T., \& Picard, D. (2015). Further Evidence for Infants' Preference for Prosocial Over Antisocial Behaviors. Infancy, 20(6), 684-692. https://doi.org/10.1111/infa.12095

Sheskin, M., Chevallier, C., Lambert, S., \& Baumard, N. (2014). Life-history theory explains childhood moral development. Trends in cognitive sciences, 18(12), 613-615.

Smith, C. L., \& Bell, M. A. (2010). Stability in infant frontal asymmetry as a predictor of toddlerhood internalizing and externalizing behaviors. Developmental Psychobiology: The Journal of the International Society for Developmental Psychobiology, 52(2), 158167.

Steckler, C. M., Liberman, Z., Van de Vondervoort, J. W., Slevinsky, J., Le, D. T., \& Hamlin, J. K. (2018). Feeling out a link between feeling and infant sociomoral evaluation. British journal of developmental psychology, 36(3), 482-500. https://doi.org/10.1111/bjdp.12232

Stets, M., Stahl, D., \& Reid, V. M. (2012). A meta-analysis investigating factors underlying attrition rates in infant ERP studies. Developmental neuropsychology, 37(3), 226-252.

Tan, E., \& Hamlin, J. K. (2021). Mechanisms of social evaluation in infancy: A preregistered exploration of infants' eye-movement and pupillary responses to prosocial and antisocial events. Infancy. https://doi.org/10.1111/infa.12447

Thibodeau, R., Jorgensen, R. S., \& Kim, S. (2006). Depression, anxiety, and resting frontal EEG asymmetry: a meta-analytic review. Journal of abnormal psychology, 115(4), 715-729. 
Woo, B. M., Steckler, C. M., Le, D. T., \& Hamlin, J. K. (2017). Social evaluation of intentional, truly accidental, and negligently accidental helpers and harmers by 10-month-old infants. Cognition, 168, 154-163. https://doi.org/10.1016/j.cognition.2017.06.029

Young, L., \& Dungan, J. (2012). Where in the brain is morality? Everywhere and maybe nowhere. Social neuroscience, 7(1), 1-10. 


\section{Preregistration}

$$
\text { https://osf.io/ht4zu/?view_only=0c4bdbc3411e4da4a2f9a5692e8f0984 }
$$

\section{Deviations from preregistration}

2.1 Number of participants. We planned to recruit 32 participants for each age group, but ended up with 10 additional participants for the 6-month group because we kept recruiting until we felt that we had recruited enough for both age groups. Because 12-month-old infants were harder to recruit, by the time we stopped recruiting for the whole project we had more participants in the 6-month group. The decision to include these participants was made prior to data analysis.

2.2 Inclusion criterion. According to the preregistered inclusion criteria, participants in the final sample should provide at least 10 artifact-free trials for helper and hinderer images, respectively. One 12-month-old infant in the final sample met all other inclusion criteria but had only 9 artifact-free trials (i.e., one trial short) for hinderer images. Due to the disruption of (further) data collection caused by COVID-19, we decided to include this participant in our data analysis. This decision was made prior to statistical analysis, and the inclusion/exclusion of this participant did not change the results of the current study.

2.3 Event timeline. We slightly adjusted the timeline of helping/hindering events based on more accurate measures of event timestamps in the videos.

2.4 The MADE pipeline. We decided to use the Maryland analysis of developmental EEG (MADE) pipeline, a standard automated preprocessing pipeline designed for the analysis of pediatric EEG data (Debnath et al., 2020), in order to maximize the removal of environmental 
noise and physiological artifacts while preserving greater amounts of EEG signal. We note that the MADE pipeline was published after we finished data collection, so the decision to incorporate this pipeline was made after preliminary analysis of EEG data. Because the MADE pipeline allows for complete automation of EEG preprocessing, and because we used the default parameters (e.g., filter parameters) of the pipeline, we believe that the incorporation of this pipeline did not inflate researcher degrees of freedom.

2.5 N290. We included the N290 component in our ERP analysis as a supplementary measure of socially relevant processes. The N290 is thought to be topographically and functionally related to the P400 component and is sensitive to social stimuli such as faces (for review, see Csibra et al., 2008; de Haan et al., 2003). We did not include this measure in the preregistration because it was not included as a neural signature in Gredebäck et al. (2015). We note that the conclusions of the current study do not hinge on the inclusion of this component; the conditional differences observed in the N290 component were also observed in the P400 component.

\section{Supplementary analyses}

3.1 Alpha asymmetry at parietal channels. To examine whether the alpha asymmetry effect was specific to frontal channels, we assessed alpha asymmetry at parietal channels. To compute parietal alpha asymmetry scores, average power density for left (P3/E28) and right (P4/E42) parietal channels in the 5-8 Hz frequency band was natural-log transformed, and difference scores $(\ln [\mathrm{P} 4]-\ln [\mathrm{P} 3])$ were calculated. A mixed ANOVA with condition (helping, hindering) and phase (Baseline, Pre, During, Post) as within-subjects factors, and age group (6month, 12-month) as a between-subjects factor found a significant interaction between age and 
phase, $F(2.71,195.17)=5.48, p=.002, \eta_{p}{ }^{2}=.071$, but no significant main effects or interactions with condition, $p \mathrm{~s}>.169$. These findings provide evidence for the specificity of alpha asymmetry effect at frontal channels.

\subsection{Alternative time window for P400 at 12 months. Because the preregistered time} window for the P400 component (250-400 ms) did not capture the P400 peak in 12-month-olds, we performed a supplementary analysis using an alternative time window (250-600 $\mathrm{ms})$ for 12 month-olds based on visual inspection of the grand-average waveform (see figure below).

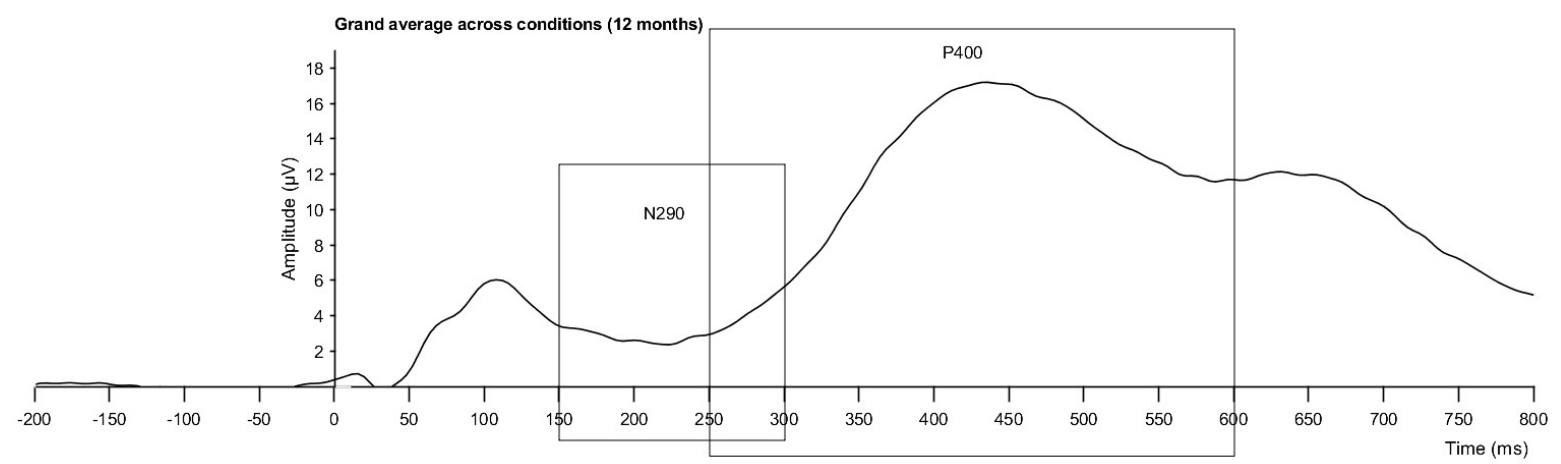

The results were similar to those found in the preregistered analysis. A 2 (condition: helping, hindering $) \times 2$ (hemisphere: left, right $) \times 2$ (age group: 6-month, 12-month) mixed ANOVA found a main effect of condition, $F(1,70)=4.16, p=.045, \eta^{2}=.06$, a main effect of age, $F(1,70)=28.65, p<.001, \eta^{2}=.29$, and a main effect of hemisphere $F(1,70)=11.04, p=.001$, $\eta^{2}=.14$. No significant interactions were found, $p \mathrm{~s}>.05$. Specifically, $\mathrm{P} 400$ mean amplitude was higher for hinderer $(M=7.47,95 \%$ CI [5.35 9.60]) than helper ( $M=5.48,95 \%$ CI $[3.577 .38])$ images, higher for 12-month-olds ( $M=11.21,95 \% \mathrm{CI}$ [8.55 13.87]) than 6-month-olds ( $M=1.74$, $95 \% \mathrm{CI}[-0.584 .05])$, and higher for the right hemisphere ( $M=7.65,95 \% \mathrm{CI}[5.699 .61])$ than the 
left hemisphere ( $M=5.30,95 \%$ CI [3.46 7.13]). These findings provide further evidence for the robustness of the $\mathrm{P} 400$ effect across age groups.

3.3 P400, Nc, and N290 analyses including number of images viewed as a covariate. To explore whether the P400, Nc, and N290 effects were affected by the number of helper/hinderer images viewed by infants, we performed analyses of covariance (ANCOVAs) including number of helper/hinderer images viewed as a covariate. The results were similar to those found in the original ANOVA analyses. For the P400 component, there were a main effect of condition, $F(1$, $69)=4.76, p=.033, \eta^{2}=.07$, a main effect of age, $F(1,69)=14.20, p<.001, \eta^{2}=.17$, and a main effect of hemisphere $F(1,69)=10.19, p=.002, \eta^{2}=.13$. For the Nc component, there was a main effect of age, $F(1,69)=28.47, p<.001, \eta^{2}=.29$ but no main effect or interactions for condition, $p \mathrm{~s}>.05$. For the $\mathrm{N} 290$ component, there were a significant main effect of age group, $F(1$, $70)=7.93, p=.006, \eta^{2}=.10$, and a marginally significant main effect of condition, $F(1,70)=3.94$, $p=.051, \eta^{2}=.05$ The directions of these effects were the same as those found in the original ANOVA analyses.

\subsection{Correlations between frontal alpha asymmetry and N290/P400. As an exploratory} analysis, we examined whether frontal alpha asymmetry difference scores (helping - hindering) in the During- and Post-phases of familiarization trials predicted P400, N290, and Nc difference scores (helping - hindering) during test trials. Results found no significant correlations between these measures in either 6- and 12-month-old infants, $p \mathrm{~s}>.05$. These findings suggest that infants' approach-avoidance responses during helping/hindering events were relatively independent of their neural responses to still images of the helper and hinderer. 